\title{
Investigations on the development of the permeability properties of binary blended concrete with nano-SiO2 particles.
}

\begin{abstract}
Water permeability of cement-based concrete has been recognized as a critical intrinsic property highly affecting the durability of reinforced concrete. An experimental study was done, designed to examine the water permeability and setting time of Portland cement mortar with nano-SiO2 admixed at $0.5,1,1.5$, and $2 \mathrm{wt} \%$ of cement. The percentage, velocity, and coefficient of water absorption tests results showed that the incorporation of nano-SiO2 particles improved the water penetration resistance of the binary-blended concrete. Such improvements were especially significant when using $2 \mathrm{wt} \%$ of nano-SiO2. The experimental results revealed that the admixing of nano-SiO2 particles not only led to denser cement mortar but also changed the morphology of cement hydration products. Mechanisms were proposed to explain the physicochemical changes induced by the nano-SiO2 particles and the specific surface area of them is demonstrated as one of the key factors. Considering the higher strength and durability is promising their use in binary-blended concrete.
\end{abstract}

Keyword: Nano-SiO2 particles; Binary-blended concrete; Permeability; Setting time 